\title{
La noción de convención social. Una aproximación analítica ${ }^{1}$
}

\section{Luis Miguel Miller Moya}

Centre for Experimental Social Sciences

Nuffield College. University of Oxford

New Road OX1 1NF. Oxford. UK

luis.miller@nuffield.ox.ac.uk

\section{Resumen}

El objetivo de este artículo es la integración de las nociones económica y sociológica de convención social en una única definición analítica. Para ello, se comienza estableciendo una distinción entre la noción de convención predominante en la teoría económica, o convención de comportamiento, y la noción sociológica de convención, o convención normativa. La primera es definida como una regularidad social arbitraria, indeterminada y estable, mientras que la última es definida como un principio de acción que prescribe cómo comportarse en determinadas situaciones. Tras desarrollar ambos conceptos, éstos son integrados en una definición dual de convención social. El trabajo concluye con una tipología de convenciones sociales derivada de la definición propuesta.

Palabras clave: sociología analítica, convenciones sociales, teoría económica, teoría sociológica.

\section{Abstract. The notion of social convention. An analytical approach}

This paper aims to integrate both economic and sociological notions of convention in a single analytical definition. To this end, it starts by distinguishing conceptually between the prevailing notion of convention in economic theory, or behavioral convention, and the sociological notion of convention, or normative convention. The former is defined as an arbitrary, indeterminate, and stable social regularity, whereas the latter is defined as a principle of action prescribing how to behave in certain situations. After developing both concepts, they are integrated in a dual definition of social convention. The paper concludes presenting a typology of social conventions derived from the proposed definition.

Key words: analytical sociology, social conventions, economic theory, sociological theory.

1. Este trabajo se ha beneficiado de las críticas y los comentarios de Fernando Aguiar, Pablo Brañas, Andrés de Francisco, Francisco Herreros, Ana León, José María Maravall, José Antonio Noguera e Ignacio Sánchez Cuenca. Del mismo modo, se ha beneficiado de la concesión de un Proyecto I+D financiado por el Ministerio de Educación y Ciencia y el FEDER, con referencia SEJ2006-00959/SOCI. Obviamente, la responsabilidad sobre el contenido del texto es enteramente mía. 


\section{Sumario}

Introducción

La teoría económica y las convenciones de comportamiento

La teoría sociológica $\mathrm{y}$ las convenciones normativas
Una definición integrada de convención social

Conclusiones

Referencias bibliográficas

\section{Introducción}

No podríamos hablar de sociedad sin convenciones sociales. Sin ellas, los humanos nos sentiríamos perdidos en toda interacción social, sin saber cómo actuar en cada encuentro con los demás, desbordados por la necesidad de reinventar cada pauta de comportamiento en la sociedad. Así, resulta imposible imaginar un contrato de compraventa sin medio de intercambio alguno, como el papel moneda, o una celebración — digamos una boda - en la que los participantes no conocieran la indumentaria que han de llevar. Difícil sería emprender cualquier búsqueda en un diccionario o en una enciclopedia, si no existiese un modo de ordenar los volúmenes o si las palabras estuvieran dispersas en lugar de seguir un abecedario. Tan obvia nos resulta la existencia de convenciones sociales, que rara vez caemos en la cuenta de nuestra experiencia cotidiana con ellas ${ }^{2}$. Asumimos que existen sin preguntarnos acerca de las condiciones de su existencia; sin cuestionarnos, además, si existen otras alternativas a las mismas. Pero lo cierto es que sería difícil escapar de una convención sin caer en otra o sin caer en otro marco regulativo que nos ayude a desenvolvernos en nuestra sociedad.

A pesar de este contacto diario con las convenciones sociales, no resulta tan sencillo el hecho de explicarlas desde un punto de vista teórico. Como trataré de hacer evidente en este artículo, explicar la emergencia, la evolución y el ocaso de las convenciones sociales ha sido un objetivo perseguido por un buen número de filósofos y científicos sociales ${ }^{3}$ sin que, por el momento, dispongamos de una teoría que haya cerrado el debate acerca de qué son y por qué

2. En realidad, normalmente sólo nos damos cuenta de que nos hallamos en presencia de una convención social cuando nuestras creencias o nuestro comportamiento chocan contra ésta. La literatura está plagada de ejemplos en este sentido. Por citar sólo uno bastante revelador, he elegido el siguiente fragmento de Lolita, la universal obra de Nabokov, en la que el protagonista, Humbert Humbert, se refiere del siguiente modo a su esposa: «Charlotte, que no se daba cuenta de la intrínseca falsedad de las convenciones y normas de conducta a las que se atenía» (Nabokov, 2002: 105).

3. Desde clásicos del pensamiento filosófico, como David Hume, hasta filósofos analíticos de la segunda mitad del siglo XX, como David Lewis, Edna Ullmann-Margalit o Margaret Gilbert; desde clásicos de la teoría sociológica, como Ferdinand Tönnies o Max Weber, hasta sociólogos contemporáneos, como los pertenecientes a la escuela francesa de la Economía de las Convenciones; desde clásicos del pensamiento económico, como John Maynard Keynes o Thorstein Veblen, hasta teóricos de juegos contemporáneos, como Ken Binmore o Robert Sugden. 
existen. En este trabajo, pretendo realizar una pequeña aportación a dicha discusión. Intentaré mostrar el camino seguido por distintos teóricos sociales para desvelar los interrogantes que nunca nos planteamos en nuestra experiencia cotidiana con las convenciones sociales.

Como punto de partida, diré que una convención es un tipo específico de institución humana ${ }^{4}$. En otras palabras, dentro del conjunto más amplio de las instituciones humanas se encuentra el subconjunto de las convenciones sociales. Si bien esta primera observación podría ser aceptada por la mayoría de teóricos sociales, la cuestión de qué características definen el subconjunto de las convenciones sociales no parece tan clara. A lo largo de este ensayo tendremos la oportunidad de contrastar buena parte de las teorías contemporáneas de las convenciones sociales. Sin embargo, antes de esto, y ya que he definido la convención como un tipo específico de institución, parece lógico comenzar la argumentación con una breve exposición acerca de qué es una institución.

En un artículo seminal, Crawford y E. Ostrom (1995: 582) afirman que «las instituciones son regularidades duraderas en la acción humana en situaciones estructuradas por reglas, normas y estrategias compartidas, así como por el mundo físico». Del trabajo de ambas autoras se deduce que en toda institución pueden ser distinguidos, al menos desde un punto de vista analítico, dos componentes: un componente de regularidad duradera y estable en el comportamiento humano y un componente estructurante ${ }^{5}$, constituyente o normativo que, a modo de prescripción, «ordena» dicha regularidad de comportamiento.

Esta consideración de la "doble naturaleza» de las instituciones, tan clara en el planteamiento de Elinor Ostrom (Crawford y E. Ostrom, 1995; E. Ostrom, 2005), no puede decirse que haya calado hondo en las propuestas teóricas en torno a dicho tema procedentes de las distintas ciencias sociales. Muy al contrario, ante la pregunta acerca de qué es una institución, diversas corrientes teóricas han aportado respuestas diferentes, a veces contrapuestas. Sin embargo, a pesar de ello, y gracias al trabajo de Crawford y E. Ostrom (1995) mencionado anteriormente, es posible agrupar tales propuestas en torno a dos grandes bloques.

El primero de estos bloques es el que considera las instituciones como equilibrios sociales; basado en las propuestas teóricas de autores como Menger (1963) o Hayek $(1945,1967)$ y en elaboraciones más recientes como las de Riker (1980), Schotter (1981) o Sugden (1986). Este primer grupo de propuestas, cuya influencia sobre la teoría económica de las instituciones ha sido determinante, pone el énfasis en el componente de regularidad de comportamien-

4. En la segunda mitad del siglo pasado asistimos a un renacimiento de propuestas de marcos teóricos integrados que pretenden contribuir a resolver la cuestión de la emergencia, la evolución y el mantenimiento de las instituciones sociales. Ver, entre otros, Schotter (1981), Williamson (1985), North (1990), Hechter, Opp y Wippler (1990), Ostrom (1990) o Knight y Sened (1995).

5. La noción de institución que aquí empleo es bastante similar a la noción de «sistema» empleada por Giddens (1979; 1984). 
to presente en toda institución humana y, de forma muy resumida, entiende ésta como una pauta estable de comportamiento en sociedad. El segundo grupo de propuestas es más heterogéneo e incluye a dos subgrupos a los que Crawford y E. Ostrom (1995: 582) han denominado «enfoque de las instituciones-comonormas» y "enfoque de las instituciones-como-reglas». El primero de ellos incluye a autores como Lewis (1969), Ullmann-Margalit (1977) o Coleman (1987, 1990a, 1990b), mientras que el segundo puede encontrarse en los trabajos de V. Ostrom (1980, 1987, 1991), Willianson (1985), North (1990) o E. Ostrom (1986, 1990). A pesar de las diferencias entre estos dos subgrupos, ambas aproximaciones «se centran en los constreñimientos lingüísticos (hablados, escritos o prescripciones o consejos entendidos tácitamente) que influyen de forma conjunta sobre las preferencias y el comportamiento optimizador de los actores» (Crawford y E. Ostrom, 1995: 582).

De un modo similar a lo ocurrido con el concepto de institución social, también las propuestas teóricas acerca de la noción de convención social pueden ser encuadradas en torno a dos grupos de aproximaciones teóricas. Un primer grupo, predominante en la teoría económica contemporánea, considera las convenciones sociales como regularidades arbitrarias y estables en el comportamiento humano. Debido a la prominencia del componente conductual en este tipo de explicaciones, en lo que sigue denominaré a la noción de convención resultante convención de comportamiento. Una segunda aproximación, anclada en los fundamentos metodológicos de clásicos de la sociología como Ferdinand Tönnies (1907) o Max Weber $(1964)^{6}$, hace hincapié en el componente normativo de las convenciones sociales y, por tanto, me referiré a la misma como convención normativa.

En este artículo, me ocuparé de caracterizar ambas propuestas teóricas por separado para, en un último punto antes de las conclusiones, especular sobre la posibilidad de una integración teórica de ambas propuestas.

\section{La teoría económica y las convenciones de comportamiento}

Pensemos en el siguiente ejemplo. Cuando tomamos el autobús o el metro, entramos en un teatro o en un cine donde los asientos no están numerados o intentamos comprar algo en una tienda abarrotada de clientes, generalmente seguimos una pauta de comportamiento en la que la prioridad sobre recursos escasos es asignada según el orden de llegada. Así, por ejemplo, si el autobús dispone de veinte asientos, sólo los veinte primeros viajeros que accedan al mismo se sentarán ${ }^{7}$. Dicha pauta conductual, o convención de comportamiento en la

6. En realidad, las referencias contemporáneas a una noción normativa de convención citan predominantemente a Weber, y éste a su vez hace referencia a Tönnies como un antecedente de su noción de convención social.

7. Esto será así siempre que la convención de comportamiento mencionada no entre en conflicto con otras convenciones alternativas, tales como "ceder el asiento a una persona mayor», "ceder el asiento a una mujer embarazada», etc. 
terminología que empleo en este trabajo, ha despertado el interés teórico de un buen número de filósofos analíticos y teóricos económicos en las cuatro últimas décadas. Sin embargo, la historia de la noción conductual de convención es algo más antigua. La gran mayoría de estos autores citan el antecedente de la noción de convención propuesta por el filosofo de la ilustración escocesa David Hume (1978 [1740], 1975 [1777]) .

Aunque no existe un consenso pleno sobre la definición económica de convención, todos los autores que la manejan estarían de acuerdo en al menos tres de sus características ${ }^{9}$ : en primer lugar, una convención es una regularidad de hecho en el comportamiento social; en segundo lugar, una convención es una regularidad de comportamiento arbitraria y, en tercer lugar, una convención es una regularidad de comportamiento estable. A continuación, expondré brevemente a qué se refieren estos teóricos con cada una de estas características.

Una convención es una regularidad de hecho en el comportamiento de un determinado grupo debido a que supone la pauta de comportamiento realmente seguida por dicho grupo en una situación recurrente de interacción social (Lewis, 1969: 42; Schotter, 1981: 10; Young, 1996: 105). Por tanto, las convenciones son pautas de comportamiento empíricamente observables. Por ejemplo, saludar con un simple «buenos días» cuando uno llega al trabajo es la convención establecida en mi centro de trabajo, pero si me encuentro con un colega fuera del mismo, la convención es «estrechar las manos» si el encuentro se produce entre dos hombres y "dar dos besos» si una de las dos partes es una mujer. En general, todas las regularidades de comportamiento relacionadas con los «buenos modales» son consideradas convenciones, ya que suponen la regularidad de comportamiento predominante en una situación particular de interacción social. Pero las convenciones no sólo están relacionadas con la «educación» y el «civismo», sino también con muchos otros aspectos sociales y económicos. Algunos ejemplos recurrentes de convenciones incluyen: adherirse a un determinado modo de vestir, usar una palabra con su significado convencional o multitud de aspectos relacionados con los mercados y la actividad económica, tales como los diferentes medios de intercambio, los estándares industriales o las reglas de contabilidad. En todos estos casos, la convención supone una práctica habitual.

8. Ver, entre otros, Lewis (1969), Schotter (1981), Sugden (1986, 1989, 1998), Skyrms (1996), Young (1993, 1996, 1998a, 1998b) y Binmore (1994, 1998).

9. No todos los economistas estarían de acuerdo con esta afirmación y quizá necesite ser aclarada. La afirmación no tendría más problemas si sólo nos referimos a la corriente principal de la economía contemporánea (mainstream economics), fundamentada en los supuestos de la teoría de la elección racional y la teoría de juegos. Sin embargo, Latsis (2004, 2005, 2006) se refiere al precedente en el uso de una noción de convención bien distinta por autores como Keynes (1973: capítulo 12) o Veblen. El uso que estos clásicos del pensamiento económico dan al concepto de convención poco tiene que ver con las tres características presentadas a continuación. Para una interpretación reciente de la noción de convención en Keynes, ver Bibow, Lewis y Runde (2005). 
En segundo lugar, los teóricos económicos asumen que una convención es arbitraria, ya que supone únicamente la puesta en práctica de una de las múltiples regularidades potenciales que podrían emerger en una situación recurrente de interacción social (Lewis, 1969: 70; Coleman, 1990a: 248; Binmore, 1994: 290). Volvamos al ejemplo del autobús. En distintas sociedades y situaciones históricas, hemos podido observar diversas soluciones a un problema aparentemente tan simple como repartir el número (escaso) de asientos disponibles en un autobús entre todos los viajeros dispuestos a utilizarlos. Así, podemos pensar en las convenciones racistas norteamericanas en la primera mitad del siglo XX o la convención de ceder el asiento a una mujer en sociedades cargadas de convenciones de género. Sin embargo, a la hora de evaluar esta segunda característica, planteo una salvedad al modo como la entienden los economistas. Aunque éstos en no pocas ocasiones afirman que las convenciones son completamente arbitrarias (ver, por ejemplo, Binmore, 1994: 290), creo que es más correcto sustituir esta idea de una completa arbitrariedad por lo que denomino rango limitado de arbitrariedad e indeterminación. De este modo, diré que las convenciones tienen un rango limitado de arbitrariedad en tanto que, aunque generalmente somos capaces de pensar en regularidades sociales alternativas a la convención establecida, éstas no son ilimitadas. Pensemos, por ejemplo, en las convenciones que regulan el saludo en distintas sociedades. Este rango limitado de arbitrariedad hace que el resultado del problema de interacción social en el que emergen las convenciones sea indeterminado, es decir que no pueda ser deducido directamente de la estructura interna del propio problema.

Por último, los teóricos económicos asumen que una convención es una regularidad estable de comportamiento. Aunque la mayoría de los teóricos mencionados anteriormente están de acuerdo con este enunciado, no todos lo están con el significado preciso de este criterio de estabilidad. Así, mientras autores como Lewis (1969) justifican la estabilidad de las convenciones a través de la reconstrucción del proceso de razonamiento de aquellos implicados en la situación de interacción social en la que una convención particular emer$\mathrm{ge}^{10}$, otros, como Sugden $(1986,1989)$, basan su explicación en el desarrollo de disposiciones de comportamiento a través de un proceso adaptativo, que tarde o temprano conduce a los individuos a coordinarse en una convención concreta. Esta última interpretación ha llevado a un buen número de teóricos económicos a definir una convención como una combinación de estrategias evolutivamente estables. Así, desviarse de la convención — por ejemplo: circular por la derecha en el Reino Unido- supone siempre un comportamiento subóptimo y la propia dinámica de la interacción social forzará a los individuos a conformarse con la convención ${ }^{11}$.

10. Este tipo de argumentación enfatiza la idea de que las convenciones se refuerzan a sí mismas (self-enforcing character). Para una discusión reciente en torno a dicho argumento, ver Cubitt y Sugden (2003).

11. Young (1996) argumenta cómo la convención que implica «conducir por la derecha» en el Reino Unido emergió de forma más o menos espontánea en este país en el siglo XVIII. 
A partir de la aplicación de las tres características contenidas en la definición económica de convención social — regularidad de hecho, rango limitado de arbitrariedad e indeterminación y estabilidad-, se pueden definir las condiciones mínimas para que una convención se mantenga (sea estable). Sin embargo, poco se puede decir acerca de qué convención - entre aquellas convenciones potenciales que se ajustan a la definición propuesta - acabará siendo la convención establecida. Esto remite al problema de por qué los sujetos se conforman con una convención y no con otra. Y ésta es la preocupación central de la aproximación sociológica a la noción de convención social que presentaré en el siguiente apartado.

\section{La teoría sociológica y las convenciones normativas}

En el año 1989, dos décadas después de la publicación de la influyente obra de Lewis (1969) — precursora de la noción de convención manejada por los teóricos económicos-, se produjeron dos hechos importantes en el desarrollo de la historia moderna del concepto de convención social. Por una parte, la filósofa Margaret Gilbert publicó su libro On Social Facts, cuyo capítulo sexto está dedicado a realizar una crítica de la propuesta de Lewis, en primer lugar, y a la reconstrucción de una noción de convención social anclada en los fundamentos metodológicos de la sociología de Max Weber, en segundo lugar. La referencia al concepto de convención en Weber se hace explícita en la primera página de este capítulo sexto (Gilbert, 1989: 315) ${ }^{12}$. Por otra parte, en este mismo año, un grupo de sociólogos y economistas franceses, conocidos posteriormente como la Escuela Francesa de la Economía de las Convenciones, publicaron un número monográfico de la Revue Economique sobre "Economía de las Convenciones». En éste se puede encontrar una introducción de carácter programático escrita por algunos de los autores más destacados de esta escuela, tales como: Jean-Pierre Dupuy, François Eymard-Duvernay, Oliver Favereau, André Orléan, Robert Salais y Laurent Thèvenot (Dupuy y otros, 1989) ${ }^{13}$. Este grupo de pensadores franceses comparten con Gilbert la crítica de la noción conductual de convención desarrollada por Lewis y la propuesta de un concepto de convención social más cercano al ya desarrollado por Weber $(1964)^{14}$.

Francia y Suecia, por el contrario, suponen claros ejemplos del establecimiento de la norma que prescribe "conducir por la izquierda" a partir de un decreto y no de formal convencional. Sobre la diferencia entre decreto y convención, ver Ullmann-Margalit (1977: cap. III).

12. Gilbert también hace referencia a la noción de convención en Weber en artículos anteriores a On Social Facts (ver, por ejemplo, Gilbert, 1983: 242).

13. Aunque los argumentos defendidos por diferentes autores dentro de esta corriente son variados, tres compilaciones publicadas recientemente pueden dar una imagen bastante completa de lo que esta escuela de pensamiento supone. Se trata de Batifoulier (2001), Favereau y Lazega (2002) y Orléan (2004).

14. La referencia explícita al concepto weberiano de convención no se reduce a estos autores. Por ejemplo, en un artículo reciente, Biggart y Beamish (2003) realizan un repaso a la noción sociológica de convención en el que también enlazan ésta con la propuesta de Weber. 
Como en tantos otros ejemplos, las propuestas sociológicas que pueden situarse bajo la etiqueta de convención normativa resultan bastante más heterogéneas que la noción económica de convención de comportamiento. Para reconstruir el tipo ideal de la noción sociológica de convención, seguiré principalmente la propuesta de Gilbert (1989), complementándola con la propuesta original de Weber y con otras aportaciones que se irán apuntando más adelante.

A partir de la noción de convención social definida por Gilbert (1989), una convención normativa es, en primer lugar, «un principio de acción aceptado de forma conjunta, un fiat de grupo con respecto a cómo uno tiene que actuar en determinadas situaciones» (Gilbert, 1989: 377). La aceptación de este principio, o fiat, es tácita en tanto que éste no necesita ser explícito para que sea seguido por una población concreta. Además, el principio es aceptado de forma conjunta del modo que queda expresado en la siguiente oración: «Nosotros aceptamos el principio $P$, en tanto que yo soy uno de nosotros, yo acepto el principio $P_{»}$. Un ejemplo de convención conocido por todos puede servir para ilustrar este argumento. Al referirnos a la convención existente en España de vestir de negro en un funeral, ésta podría ser formulada del siguiente modo: «dondequiera que un español se encuentre en un funeral, vestirá de negro». En tanto que yo vivo en España, dicho principio sería mi principio en la situación referida.

En segundo lugar, la fuente de obligatoriedad del principio en cuestión radica en la existencia de mecanismos de aprobación de los comportamientos que se ajustan al principio y de mecanismos de desaprobación de los comportamientos contrarios a éste. Siguiendo en este punto al propio Weber (1964), estos mecanismos pueden ir desde una mirada sutil de desaprobación hasta el «boicot declarado» (la "condena al ostracismo social») por parte de los miembros del grupo. Uno puede obtener una "prueba experimental» de esta segunda característica vistiendo de rojo en el próximo funeral al que asista ${ }^{15}$.

En tercer lugar, para Gilbert (1989: 367), aunque no son lo mismo, «existe una analogía llamativa entre el "deber" de una convención social [...] y el "deber" de un acuerdo explícito». En ambos casos, uno no puede decidir individualmente que un acuerdo o una convención dejan de tener validez, ni puede establecer unilateralmente un nuevo acuerdo o convención. En este sentido, ambos conceptos tienen un carácter colectivo. Por ejemplo, aunque considere que vestir formalmente no es necesario para llevar a cabo mi trabajo diario, yo

15. Es preciso aclarar que Gilbert (1989) no incorpora esta segunda característica a su definición de convención social. Para la filósofa analítica norteamericana, la normatividad intrínseca presente en todo principio — como argumentaré más adelante- es suficiente. Sin embargo, parece obvio que si un principio, por el mero hecho de existir, genera normatividad, los participantes en el mismo se sentirán obligados a recriminar a aquéllos que se desvían del mismo. Y dado que ésta es la base de la noción weberiana de convención, he considerado pertinente incluir esta segunda característica en la caracterización de la noción sociológica de convención. 
no puedo establecer por mí mismo que la convención que prescribe vestir formalmente deja de existir en mi centro de trabajo.

Por último, aunque una convención puede basarse en razones morales, el carácter normativo de las convenciones no tiene por qué coincidir desde un punto de vista lógico con el carácter normativo de las normas morales (Weber, 1964). Así, mientras las normas morales distinguen entre «buenas» y «malas» acciones, las convenciones normativas «proporcionan una base para el juicio acerca de lo apropiados que son los actos propios y ajenos» (Biggart y Beamish, 2003: 444). Dado que he definido una convención normativa como un principio general de acción, la normatividad contenida en ésta puede ser explicada del mismo modo que Gilbert lo hace para los principios: «en buena lógica, si esto o lo otro es tu principio, entonces, desde tu propio punto de vista, tienes una razón para conformarte» (Gilbert, 1989: 374). Y dicha conformidad es independiente de posibles argumentos morales. Pensemos en la convención ya referida de vestir de negro en un funeral. Para que definiéramos dicho principio como un principio moral, deberíamos poder decir algo acerca de la razón última que cada individuo tiene para seguir dicho principio. Sin embargo, a poco que indaguemos sobre la multiplicidad de motivaciones individuales que pueden conducir a dicho comportamiento social, nos damos cuenta que «vestir de negro en un funeral» no está obligatoriamente ligado a un valor intrínseco $(\text { value })^{16}$. Por ejemplo, uno puede seguir la convención «vestir de negro en un funeral» tanto si considera que dicha convención es buena intrínsecamente, como si piensa todo lo contrario. En el primero de los casos, tiene una motivación intrínseca para seguir la convención; en el segundo, puede seguirla simplemente por imitación, anticipando la desaprobación de las personas que le rodean o por cualquier otro motivo.

Las cuatro características presentadas establecen una base para explorar el carácter normativo de las convenciones sociales y, como argumentaré en el siguiente apartado, suponen un complemento necesario para la noción económica de convención.

\section{Una definición integrada de convención social}

Tanto Weber (1964: 264) como, más recientemente, Kliemt (1986: 169), han puesto de manifiesto el peligro de confundir las regularidades de hecho que observamos en la conducta humana con el conjunto de principios que contribuyen a la regulación normativa de dicha conducta. En otras palabras, no podemos olvidar el carácter «bifronte» de las convenciones sociales ${ }^{17}$. Por ello, cualquier definición del concepto de convención social debería responder al

16. Utilizo aquí el término "valor intrínseco» (value) en el sentido empleado tradicionalmente por la sociología, es decir, como una creencia compartida de lo que es considerado «bueno/deseable» $\mathrm{o}$ «malo/indeseable» en el seno de una sociedad determinada.

17. Es importante recordar la definición de institución social de E. Ostrom y Crawford (1995) presentada en la introducción de este trabajo. 
menos a las dos preguntas siguientes: 1) ¿Qué características definen el tipo de regularidad de hecho al que denominamos convención social? 2) ¿Por qué los sujetos individuales ajustan su comportamiento a dicha regularidad? Si la definición presentada a continuación responde satisfactoriamente a ambas preguntas, se puede afirmar con cautela que disponemos de una definición provisional que da cuenta de lo que intuitivamente denominamos convención social. Sin más, defino convención social como:

Una regularidad $R$ empíricamente observable en el comportamiento humano que cumple las siguientes características: 1 ) se trata de una regularidad $R$ arbitraria e indeterminada, en tanto que podría ser identificada una regularidad alternativa $R$ ' que respondiera de igual forma a la demanda originada por la estructura de la situación de interacción social a partir de la cual ha surgido $R ; 2)$ se trata de una regularidad estable, cuya estabilidad puede deberse a dos motivos: bien los individuos no tienen incentivos para desviarse de la misma (problemas puros de coordinación en los que existe una coincidencia plena de intereses entre los participantes) o bien cualquier desviación de la convención está penalizada por la desaprobación de aquéllos que participan en ella, ya que para éstos dicha desviación no supone sólo comportarse de forma diferente a la regularidad $R$, sino que supone desviarse de un principio normativo. Ambos motivos no son excluyentes ${ }^{18}$.

Aunque analíticamente resulte necesario separarlos, hay que decir que regularidad y principio no son entidades independientes, sino que forman parte de la misma noción de convención ${ }^{19}$.

Tras hacer explícita mi definición de convención social, es preciso preguntarse si ésta es capaz de responder a las dos preguntas lanzadas más arriba - la primera acerca de las características que definen el tipo de regularidad de hecho al que denominamos convención social y la segunda acerca de las razones que llevan a los sujetos a ajustar su comportamiento a dicha regularidad. La respuesta sería un sí, al menos un sí provisional. En primer lugar, a través de las características de arbitrariedad y estabilidad podemos distinguir las convenciones sociales de otras regularidades empíricamente observables en el com-

18. Nótese que, en esta definición, he empleado sólo una de las estrategias posibles para captar el carácter dual de las convenciones sociales. En concreto, siguiendo la noción de principio en Gilbert (1989), he incluido en la definición esta idea de principio como una motivación individual distinta a la maximización del propio interés propia de las explicaciones económicas.

19. Esta «doble naturaleza» de las convenciones ha sido puesta de manifiesto, empleando términos distintos, por otros autores, especialmente algunos de los integrantes de la Escuela Francesa de la Economía de las Convenciones. Por ejemplo, Favereau (1986) habla de "Convención ${ }_{1}$ "

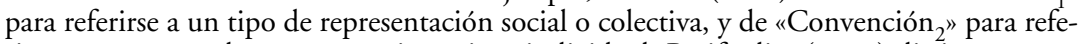
rirse a una pauta de comportamiento interindividual; Batifoulier (2001) distingue entre "modos convencionales de evaluación», para referirse a "convenciones de pensamiento», y «reglas convencionales», para referirse a "convenciones de comportamiento»; Orléan (1999) utiliza la noción de "convención de interpretación» para referirse al componente evaluativo o normativo de las convenciones. 
Tabla 1. Tipología de convenciones

\begin{tabular}{|c|c|c|}
\hline & Principio difuso & Principio bien definido \\
\hline Regularidad muy extendida & $\begin{array}{l}\text { Convenciones } \\
\text { emergentes }\end{array}$ & $\begin{array}{l}\text { Convenciones } \\
\text { establecidas }\end{array}$ \\
\hline Regularidad poco extendida & $\begin{array}{l}\text { Convenciones } \\
\text { difusas }\end{array}$ & $\begin{array}{l}\text { Convenciones } \\
\text { decadentes }\end{array}$ \\
\hline
\end{tabular}

portamiento humano. Siguiendo de nuevo a Weber, las convenciones no pueden ser clasificadas como costumbres, ya que en éstas últimas falta la aprobación y la desaprobación del resto. Las convenciones son distintas a lo que denominamos moda, en tanto que ésta última es efímera por definición y no estable como las convenciones. Las convenciones pueden coincidir con las situaciones de intereses, aunque sólo en un escenario de condiciones muy concreto: cuando nos hallamos ante problemas puros de coordinación (coincidencia de intereses $)^{20}$. Por tanto, estoy en condiciones de afirmar que he respondido satisfactoriamente a la primera de las preguntas planteadas. En cuanto a la segunda pregunta, relativa al carácter normativo de la convención, he identificado dos mecanismos que actúan como explicaciones plausibles del porqué de la aceptación de las convenciones. El mecanismo más general señala la normatividad intrínseca a todo principio como principal estímulo de la conformidad con la convención. Un principio que no sólo proporciona una motivación (normativa) individual — como señala Gilbert (1989)—, sino que también posibilita la labor de control de las acciones individuales por parte de los miembros de la población objeto del principio — como ya señalara Weber (1964) y, más recientemente, Biggart y Beamish (2003). En situaciones muy concretas — problemas puros de coordinación-, la función cognitiva de las convenciones sociales es suficiente ${ }^{21}$.

Como última defensa de la definición dual de convención social propuesta presentaré la tabla 1, en la que se puede observar cómo el mayor o menor peso de las características de regularidad y de principio en la convención nos permite derivar una tipología de convenciones sociales cuyo poder explicativo es

20. A las diferencias existentes entre la noción de convención social y otras regularidades de comportamiento, habría que añadir la diferencia de la misma con otro tipo de instituciones humanas, como la diferencia con las normas morales a la que me he referido con anterioridad o la diferencia con las normas legales. En contraste con éstas últimas, en las convenciones falta el "cuadro especializado de personas» encargadas de hacer cumplir las leyes (Weber, 1964).

21. Por función cognitiva me refiero a cómo las convenciones sociales, en situaciones de coincidencia de intereses, suponen un mecanismo de coordinación de las expectativas individuales. Por ejemplo, en España yo conduzco por la derecha (acción), espero que el resto conduzca por la derecha (expectativa de primer orden) y espero que el resto espere que todo el mundo conduzca por la derecha (expectativa de segundo orden). Para una aproximación formal a esta función cognitiva, ver Sacconi y Moretti (2002). 
mayor del que se derivaría de una definición unidimensional de la noción de convención social.

Así, denomino convenciones emergentes a aquéllas que responden a situaciones donde se empieza a extender una regularidad de comportamiento en el seno de una población aunque todavía no está claro el principio normativo, es decir, no está clara cuál es la conducta prescrita en una situación recurrente de interacción social. Podemos encontrar multitud de ejemplos cotidianos en situaciones novedosas, como los nuevos usos del lenguaje en el ámbito de las nuevas tecnologías (mensajes SMS, correos electrónicos, chats, etc.).

Las convenciones establecidas son aquéllas en las que podemos identificar el principio que guía la acción de forma precisa y donde la regularidad de comportamiento definida por tal principio está suficientemente extendida. Una característica interesante de las convenciones establecidas es que, a menudo, las personas tienden a olvidar el carácter arbitrario de las mismas y las ven como totalmente necesarias. Con respecto a la convención que prescribe conducir por la izquierda en el Reino Unido, Binmore (1994: 290) afirma que «algunos creen que "conducir por la derecha", más que arbitrario, es perverso. Los británicos, por ejemplo, dicen que es razonable conducir por la izquierda porque los diestros pueden mantener la mano y el brazo que dominan más en el volante mientras usan la zurda para cambiar de marcha».

Las convenciones difusas representan normalmente situaciones de transición donde ni está claro el principio predominante en un momento determinado, ni se observa una regularidad que se impone sobre el resto. Por ejemplo, un colega inglés me señalaba que, una vez que la convención que supone «estrechar la mano» de modo formal ha caído en desuso entre los jóvenes británicos, y no habiéndose incorporado una práctica distinta, éstos no saben muy bien qué fórmula emplear al saludar a otra persona. Un caso parecido ocurre cuando el encuentro se produce entre dos personas de nacionalidad distinta, donde existen principios que prescriben comportamientos distintos en lo que al saludo se refiere. Un tercer ejemplo de convención difusa lo encontramos en sociedades multilingües en las que cualquier conversación comienza con un problema de coordinación previo para determinar qué idioma va a ser empleado en la misma ${ }^{22}$.

Por último, las convenciones decadentes responden a situaciones donde, aunque está bien definido el principio que guía la conducta, dicha práctica está en claro proceso de transformación o desaparición. En países como España, éste es el caso de las convenciones que implican comportamientos diferenciados por género, tales como la convención que prescribe «servir primero a las mujeres en la mesa», "ceder el paso a una mujer al atravesar una puerta», etc.

22. Un caso extremo de los problemas de coordinación presentes en una sociedad multilingüe lo encontramos en Sudáfrica, donde, en la actualidad, conviven once lenguas oficiales. 


\section{Conclusiones}

Este trabajo comenzaba poniendo de relieve una llamativa laguna en la investigación social contemporánea: aunque es imposible concebir una sociedad sin convenciones, las distintas ciencias sociales han sido incapaces de ponerse de acuerdo sobre las características que definen a éstas, así como los mecanismos que posibilitan su existencia. En este sentido, el objetivo principal de este artículo ha sido establecer una definición provisional del concepto de convención social que pueda servir como base para investigaciones teóricas y empíricas posteriores. En el proceso de construcción de tal definición, he planteado otras cuestiones, tales como la diferencia entre la noción de convención empleada por los teóricos económicos y aquélla manejada por los sociólogos; la diferencia entre la noción de convención y otros conceptos, como los de costumbre, moda, situación de intereses, norma moral o norma legal; o el establecimiento de una tipología de convenciones en relación con la mayor o menor presencia de los componentes de la definición de convención propuesta.

La principal conclusión alcanzada ha sido constatar que una definición analítica de convención social requiere de una naturaleza dual, un carácter bifronte. Tal como han señalado otros autores para el caso de las instituciones (Kliemt, 1986; Crawford y E. Ostrom, 1995; E. Ostrom, 2005), las convenciones son, al mismo tiempo, tanto regularidades estables en el comportamiento social, como principios normativos que guían dicho comportamiento. Cualquier aproximación al estudio de las convenciones sociales no debería olvidar esta dualidad, si no quiere acabar presentando una imagen excesivamente simplista de uno de los conceptos más complejos que podemos encontrar en el seno de las ciencias sociales.

\section{Referencias bibliográficas}

Batifoulier, P. (2001). Théorie des conventions. París: Economica.

BIBOW, J.; LEWIS, P.; RUNDE, J. (2005). «Uncertainty, conventional behavior, and economic sociology». American Journal of Economics and Sociology, vol. 64 (2), p. 507532.

BIGGART, N. W.; BEAMISH, T. D. (2003). «The economic sociology of conventions: Habit, custom, practice, and routine in market order». Annual Review of Sociology, vol. 29, p. 443-464.

Binmore, K. (1994). Teoría de juegos. Madrid: McGraw Hill.

- (1998). Just playing. Game theory and the social contract. The MIT Press.

COLEMAN, J. S. (1987). «Norms as social capital», en: RADNITZKY, G.; BERNHOlZ, P. (eds.). Economic imperialism. Nueva York: Paragon.

- (1990a). Foundations of social theory. Cambridge (MA): Harvard University Press.

- (1990b). "On the emergence of norms». En: Hechter, M.; OpP, K. D.; Wippler, R. (eds.). Social institutions: Their emergence, maintenance and effects. Berlín: Walter de Gruyter.

Crawford, S.; Ostrom, E. (1995). «A grammar of institutions». American Political Science Review, vol. 89 (3), p. 582-600. 
Cubitt, R. P.; Sugden, R. (2003). «Common knowledge, salience and convention: A reconstruction of David Lewis' game theory». Economics and Philosophy, vol. 19, p. $175-210$.

Dupuy, J. P.; Eymard-Duvernay, F.; Favereau, O.; Orléan, A.; Salais, R.; THÉVENOT, L. (1989). «Introduction». Revue Economique, vol. 2, p. 141-145. FAVEREAU, E. (1986). «La formalisation du rôle des conventions dans l'allocation des ressources». En: SALAIS, R.; THÉVENOT, L. (eds.). Le Travail Marchés, règles, conventions. París: Economica.

FAVEREAU, O.; LAZEGA, E. (2002). Conventions and structures in economic organization. Cheltenham: Edward Elgar.

GidDENS, A. (1979). Central problems in social theory. Berkeley: University of California Press.

- (1984). The constitution of society: Outline of the theory of structuration. Berkeley: University of California Press.

GilberT, M. (1983). «Notes on the concept of social convention». New Literary History, vol. 14 (2), p. 225-251.

- (1989). On social facts. Londres: Routledge.

HaYeK, F. A. von (1945). "The use of knowledge in society». American Economic Review, vol. 35, p. 519-530.

- (1967). «Notes on the evolution of rules of conduct». En: HAYEK, F. A. von. Studies in Philosophy, Politics, and Economics. Chicago: University of Chicago Press.

HeChTER, M.; Opp, K. D.; Wippler, R. (1990). Social Insitutions their Emergence, Maintenance ahd Effects. Nueva York: Aldine de Gruyter.

Hume, D. (1975 [1777]). An enquiry concerning the principles of morals. Oxford: Oxford University Press.

- (1978 [1740]). A treatise on human nature. Oxford: Oxford University Press.

KEYNES, J. M. (1973). «The general theory of employment, interest and money». En: KEYNES, J. The collected writings of John Maynard Keynes, vol. VI. Londres: Macmillan.

KLIEMT, H. (1986). Las instituciones morales. Barcelona: Alfa.

KNIGHT, J.; SeNED, I. (eds.) (1995). Explaining Social Institutions. Ann Arbor: University of Michigan Press.

LATSIS, J. S. (2004). Theories and the arbitrary economy. Universidad de Cambridge.

- (2005). «Is there redemption for conventions?». Cambridge Journal of Economics, vol. 29, p. 709-727.

- (2006). "Convention and intersubjetivity: New developments in french economics». Journal for the Theory of Social Behavior, vol. 36 (3), p. 255-277.

LEWIS, D. (1969). Convention. A Philosophical Study. Oxford: Basil Blackwell.

Menger, K. (1963). Problems in Economic and Sociology. Urbana: University of Illinois Press.

NAвOKOV, V. (2002 [1955]). Lolita. Barcelona: Anagrama.

NORTH, D. (1990). Institutions, institutional change, and economic performance. Nueva York: Cambridge University Press.

OrLÉAN, A. (1999). Le pouvoir de la finance. París: Odile Jacob.

- (2004). Analyse économique des conventions. París: Quadrige. Presses Universitaires de France.

Ostrom, E. (1986). «An agenda for the study of institutions». Public Choice, vol. 48, p. 3-25.

- (1990). Governing the commons: The evolution of institutions for collective action. Nueva York: Cambridge University Press. 
- (2005). Understanding institutional diversity. Princeton: Princeton University Press. Ostrom, V. (1980). «Artisanship and artifact». Public Administration Review, vol. 40, p. 309-317.

- (1986). "A fallabilist's approach to norms and criteria of choice». En: KAUFMANN, F. K.; Majone, G.; Ostrom, V. (eds.). Guidance, control, and evaluation in the public sector. Berlín: Gruyter.

- (1991). The meaning of american federalism: Constituting a self-governing society. San Francisco: Institute for Contemporary Studies Press.

RIKER, W. (1980). «Implications from the disequilibrium of majority rule for the study of institutions". American Political Science Review, vol. 74, p. 432-446.

SACCONI, L.; MORETTI, S. (2002). «Fuzzy norms, default reasoning and equilibrium selection in games under unforeseen contingencies and incomplete knowledge». LIUC Papers, publicación periódica de la Universidad Carlo Cattaneo.

SCHOTTER, A. (1981). The economic theory of social institutions. Cambridge: Cambridge University Press.

SKYRMS, B. (1996). Evolution and the social contract. Cambridge: Cambridge University Press.

Sugden, R. (1986). The economics of rights, co-operation and welfare. Oxford: Basil Blackwell.

- (1989). «Spontaneous Order». Journal of Economic Perspectives, vol. 3 (4). p. 85-97.

- (1998). «Conventions». En: Newman, P. The New Palgrave Dictionary of Economics and the Law. Albany: Macmillan.

TÖNNIES (1909). Die Sitte. Frankfurt am Main: Rütten \& Loening.

Ullmann-Margalit, E. (1977). The emergence of norms. Oxford: Oxford University Press.

Weber, M. (1964 [1922]). Economía y sociedad. Madrid: Fondo de Cultura Económica.

Willianson, O. E. (1985). The economic institutions of capitalism: Firms, markets, relational contracting. Nueva York: Free Press.

YounG, H. P. (1993). «The Evolution of Conventions». Econometrica, vol. 61, p. 5784.

- (1996). «The economics of convention». Journal of Economic Perspectives, vol. 10 (2), p. 105-122.

- (1998a). Individual strategy and the social structure: An evolutionary theory of institutions. Princeton: Princeton University Press.

- (1998b). «Social norms and economic welfare». European Economic Review, vol. 42, p. 821-830. 